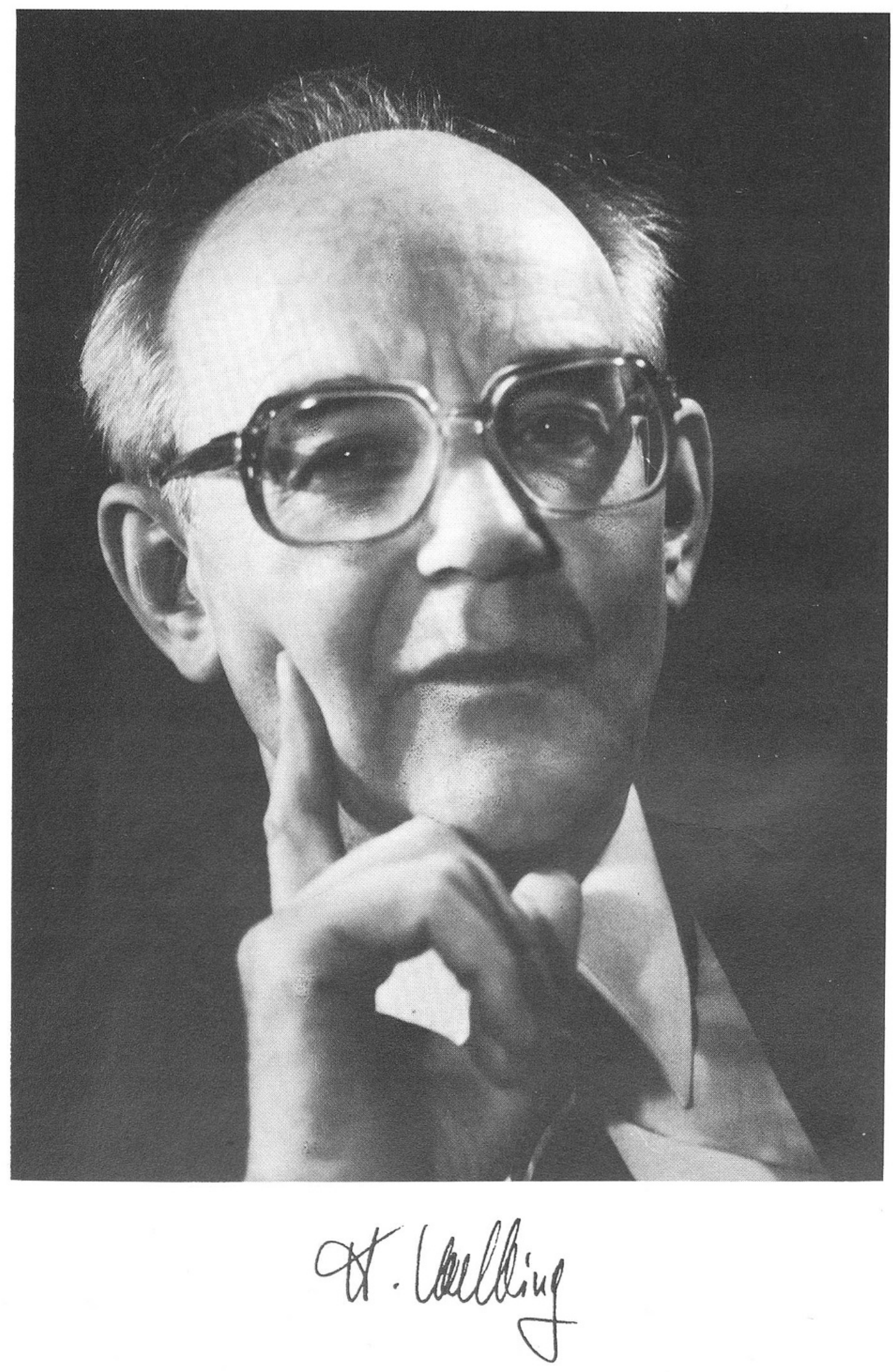




\section{Herrn Professor Dr. med. H. M. Koelbing zum 60. Geburtstag}

Am 17. Juni 1983 wird Herr Prof. Dr.med. Huldrych M. Koelbing, Ordinarius für Geschichte der Medizin und Direktor des Medizinhistorischen Instituts der Universität Zürich, 60jährig.

Huldrych M. Koelbing ist Bürger von Basel und Schinznach-Dorf (Aargau) und wurde als Sohn des Pfarrers Bernhard Koelbing in Reiden geboren. Seine Mittelschulbildung erhielt er am traditionsreichen Humanistischen Gymnasium der Stadt Basel. Von 1943 bis 1949 studierte er in Basel Medizin, verbrachte Gastsemester in Genf und Birmingham und promovierte 1950 mit einer internistischen Dissertation. Nach Assistentenjahren in Lausanne (Prof.O.Bucher) und vor allem an der Universitäts-Augenklinik Basel (Prof. F. Rintelen) ließ er sich im April 1955 als Augenarzt FMH mit einer eigenen Praxis in Basel nieder.

In der Basler Augenklinik erwachte die Freude an der Geschichte der Medizin. Ermuntert durch Prof. Rintelen, legte er 1953 seine ersten Arbeiten zur Geschichte der Augenheilkunde vor, ein Thema, dem er sich seither immer wieder gerne zuwandte. Seine weitere Entwicklung förderte die Teilnahme an den medizinhistorischen Seminarien von Prof.H.Buess, Basel, und Prof. E. H. Ackerknecht, Zürich, sowie an den Henry-E.-SigeristKonferenzen in Pura und Luzern. An der Universität Basel habilitierte er sich 1965 mit der Arbeit «Renaissance der Augenheilkunde 1540-1630» für Geschichte der Medizin. Im Jahr zuvor hatte er die Tätigkeit des Augenarztes mit der eines Redaktors der «Documenta Geigy» vertauscht. Erfolgreich brachte er in dieser vielgelesenen Publikationsreihe die historische Dimension zur Geltung.

Auf den Beginn des Wintersemesters 1971/72 wählte der Regierungsrat des Kantons Zürich Huldrych M.Koelbing als Nachfolger von Professor E.H.Ackerknecht zum ordentlichen Professor für Geschichte der Medizin und zum Direktor des Medizinhistorischen Instituts der Universität Zürich.

Seine Aufgabe formulierte Prof.Koelbing so: «Ich glaube, daß die Medizingeschichte nicht nur den Werdegang unseres Faches verstehen läßt, sondern daß sie uns auch die Orientierung in der so stürmischen Entwicklung der heutigen Medizin erleichtern kann, vor allem weil sie uns hilft, echte 
Fortschritte von den bloßen Modeströmungen, die immer wieder auftreten, zu unterscheiden. Innerhalb des medizinischen Ausbildungsprogramms hat der Medizinhistoriker m.E. die Aufgabe, den angehenden Ärzten einen Eindruck von der Medizin als Ganzem zu geben und sie gleichzeitig zu kritischem Denken anzuregen.»

Im Sinne dieses Programms versieht Herr Prof.Koelbing seit zwölf Jahren seine Lehrtätigkeit in Vorlesungen und Vorträgen, im anregenden Kreis der Seminarien und in der Kleinarbeit mit den zahlreichen Doktoranden. Es ist ihm ein lebhaftes Anliegen, über den akademischen Kreis hinaus historische Fragen in ihrer aktuellen Bedeutung, Zeitprobleme in ihrer historischen Bedingtheit der Ärzteschaft und dem gebildeten Publikum nahezubringen. Wichtige Bereiche wie die ärztliche Ethik und die medizinische Ausbildung stellt er fundiert und elegant formuliert aus dem Blickwinkel verschiedener Epochen dar, so daß das Zeitlose vor dem Wandel der Ereignisse sichtbar wird. Sein Buch «Arzt und Patient in der antiken Welt» ist bei aller gelehrten Vorarbeit und Quellenkenntnis auch ein Buch für den Patienten, für den Arzt von heute, nicht durch leichtfertige Aktualisierung, sondern durch das Aufscheinen des Immergültigen im wechselseitigen Verhältnis von Hilfsbedürftigem und Heilkundigem.

Aus dem Interesse an der Geschichte des öffentlichen Gesundheitswesens erwuchs die von Prof. Koelbing eingeleitete Zusammenarbeit seines Instituts mit Herrn Prof. M. Mattmüller und dem Historischen Seminar Basel in der Erforschung der Pest in der Schweiz. Es ist sein Verdienst, daß an den zwei bisher abgehaltenen «Schweizerischen Pest-Kolloquien» die Medizingeschichte mit den anderen Disziplinen ins Gespräch kam.

Eine Anerkennung seines Wirkens auf internationaler Ebene ist die Ernennung von Prof. Koelbing zum Mitglied des Advisory History of Medicine Panel des Wellcome Trust London im Jahr 1982.

Große und erfolgreiche Arbeit hat Herr Prof. Koelbing für die Schweizerische Gesellschaft für Geschichte der Medizin und der Naturwissenschaften geleistet. Seit 1953 Mitglied, gelangte er 1965 in den Vorstand und wurde 1969 Sekretär und Quästor; von 1977 bis 1982 leitete er die Gesellschaft als Präsident. Zwei Ziele strebte er an, beide hat er erreicht: die Suisse romande beteiligt sich vermehrt an der Arbeit der SGGMN, wie die Symposien von Neuenburg (1974) und Genf (1976) belegen; der Mitgliederbestand der SGGMN hat sich in den letzten zwölf Jahren verdoppelt.

Als Direktor des Medizinhistorischen Instituts der Universität Zürich ist Herr Prof. Koelbing ein großzügiger Chef, der seinen Mitarbeitern Ver- 
trauen und viel Entfaltungsfreiheit gewährt, ihren Anliegen stets ein offenes Ohr leiht und sie in ihrer wissenschaftlichen Entwicklung unterstützt.

Lieber Huldrych, zum 60. Geburtstag danken Dir Freunde und Leser, Doktoranden und Mitarbeiter herzlich für Deine große Arbeit, Dein Wohlwollen und die stete Förderung. Sie wünschen Dir für die kommenden Jahre Gesundheit, Wohlergehen und Verwirklichung Deiner vielfältigen wissenschaftlichen Vorhaben.

Urs Boschung

Beat Rüttimann

Verehrter Jubilar, als Gratulanten stellen sich heute auch die Autoren der Beiträge zu dieser Festschrift ein - Kollegen, Freunde, Schüler -, und ihren herzlichen Wünschen schließen sich an der Vorstand der SGGMN sowie Verlag und Redaktion des Gesnerus. 